\begin{tabular}{|l|l|l|l|l|l|}
\hline MUNIBE Antropologia-Arkeologia & $n^{\circ} 68$ & $289-300$ & DONOSTIA & 2017 & ISSN 1132-2217 • eISSN 2172-4555 \\
\hline
\end{tabular}

\title{
Estrategias cuantitativas para el estudio de cerámica arqueológica. Una propuesta desde el caso de la cerámica histórica alavesa
}

\section{Quantitative strategies for the study of archaeological pottery. A proposal from the case of historical alavese pottery}

\author{
PALABRAS CLAVES: Muestreo, Cuantificación, Cultura material, Periodo Tardo medieval, Edad Moderna Temprana. \\ GAKO-HITZAK: Sampling, Quantification, Material Culture, Late Medieval Period, Early Modern Age. \\ KEY WORDS: Laginketa, Kuantifikazioa, Kultura materiala, Ertaro beranta, Aro Berri goiztiarra.
}

\section{Sergio ESCRIBANO-RUIZ(1)}

\section{RESUMEN}

Apenas existen trabajos explícitos sobre técnicas de cuantificación aplicadas a la cerámica arqueológica publicados en castellano. Por ello, el objetivo de este artículo es proporcionar un ejemplo testado y razonado de estrategia de cuantificación diseñada para estudiar una muestra cerámica determinada. En este trabajo se abarcan el diseño estadístico conceptual, los parámetros de confección de la muestra y la discusión sobre las técnicas cuantitativas empleadas. Por un lado, tratamos de conceptualizar cómo las unidades de excavación se convierten en unidades estadísticas y proponemos un protocolo selectivo de contextos para confeccionar una muestra cerámica libre de grandes distorsiones generadas por los procesos de formación del registro arqueológico. Por otro lado, discutimos las técnicas cuantitativas utilizadas y justificamos la aportación de cada una de ellas. Finalmente, argumentamos que el procedimiento de trabajo descrito permite crear indicadores cronológicos que pueden ser extrapolables a otros trabajos y ámbitos geográficos.

\section{LABURPENA}

Nekez aurkitu daiteke kuantifikazio teknikak eta zeramika jomuga dituen erdaraz idatzitako lanik. Hau dela eta, testu honen helburua balioetsia izan den zenbaketa estrategia arrazoitu baten adibidea plazaratzea da. Kasu jakin honetan zeramika lagin baten inguruan eraikia izan dena. Lanean zehar diseinu estatistiko kontzeptuala lantzen da, lagina sortzeko parametroak azaltzen dira eta kuantifikazio teknika ezberdinen inguruan eztabaidatzen da. Zehazki, indusketa unitateak unitate estatistiko bilakatzeko prozedura deskribatu eta estratifikazioaren sortze prozesuek eragindako itxuraldaketa prozesuak txikiagotzen dituen lagin eraketa protokolo bat proposatzen dira. Aldi berean, erabiliak izan diren zenbaketa teknika ezberdinen eztabaida eta bakoitzaren ekarpenen justifikazioa burutzen ditugu. Azkenik, prozedura honen bitartez beste lan zein testuinguru geografikoetan erabiliak izan daitezkeen adierazle kronologikoak sortu daitezkeela argudiatzen da.

\begin{abstract}
There is a lack of Spanish literature explicitly concerning quantification techniques applied to archaeological pottery. That is why this paper aims to provide a tested and reasoned example of a quantification strategy designed to study a concrete pottery sample, recovered in Araba and dating back to $14^{\text {th }}$ to $17^{\text {th }}$ centuries. This work covers both the conceptual statistical design, as the parameters to create the sample and the discussion about the suitability of the different quantification techniques to be used. First we try to conceptualize how excavation units are converted into statistical units and then propose a protocol for selecting the contexts that will be part of the sample by minimizing taphonomic biases. Then we discuss the quantification techniques used in our study and justify the contribution of each of them. Finally, we argue that proposed working procedure enables creating chronological indicators ready to be used in other studies and geographical areas.

In summary, our proposal stresses the importance of studying contextualized pottery, which implies either using only pottery recovered at archaeological excavations to build a sample, either making the stratigraphic context the unit of statistic reference. We enhance that selected contexts should be as free of biases caused by the formation processes of the archaeological record as possible. In accordance we set up a selective proposal that deals with qualitative and quantitative aspects of the sample making process. Nonetheless, the core proposal of this work is a dual quantification system to answer the questions posed both by the systemic and the archaeological context of pottery. For the first aspect we propose the use of descriptive quantification techniques, in order to calculate the fragmentation index of any assemblage, and an estimative one, Minimum Number of Individuals, for the second. Last, but not least, we put forward a central question when talking about samples and sampling. Are our results significant and, so that, comparable? By means of a practical example we illustrate the significance of the sample and, then, propose a way to make it comparable to any other sample whatever its size or geographical location.
\end{abstract}

\footnotetext{
(1) Grupo de Investigación en Patrimonio Construido, GPAC (UPV/EHU) Departamento de Geografía, Prehistoria y Arqueología

C/ Tomas y Valiente s.n.

01006, Vitoria-Gasteiz

sergio.escribanor@ehu.es
} 


\section{1.- INTRODUCCIÓN}

La arqueología, entendida no tanto como el estudio de las cosas antiguas sino como la disciplina de las cosas (OLSEN et. al, 2012: 3-7), trabaja hoy sobre aspectos materiales del pasado y del presente. Los paisajes y la arquitectura son estudiados igual que el subsuelo, como palimpsestos temporales, espaciales o semióticos (BAILEY, 2007: 203-210), como documentos estratificados en los que interactúan diversas acciones humanas y naturales realizadas en diferentes momentos. La repetición constante y el dinamismo de estas interacciones genera una materialidad fragmentada, transformada y enredada. Este hecho se manifiesta especialmente en los procesos de estratificación que se nos presentan como complejos rompecabezas, cuya solución tratamos de resolver recurriendo a distintos conjuntos de técnicas. La premisa de la complejidad del registro arqueológico ha sido generalmente asumida y ha llevado a algunos autores a ahondar en los innumerables procesos de mediación entre lo que existía y lo que recuperamos (SCHIFFER, 1987), y a otros incluso a equiparar la excavación arqueológica con un descenso a los infiernos (CARANDINI, 1997: 42, 248), para reflejar la dificultad que conlleva el análisis estratigráfico (AZKARATE, 1999: 36-37). En este contexto epistemológico, generalmente se asume que nuestro punto de partida no es más que una muestra caprichosa y contingente de la realidad que pretendemos caracterizar y comprender. Consecuentemente, tal como algunos autores reconocen de forma explícita, las muestras con las que contamos en arqueología no son demasiado buenas (FERNÁNDEZ MARTíNEZ, 2015: 18).

Mediante este trabajo queremos proporcionar un ejemplo, argumentado y razonado, de tratamiento de una muestra de uno de los materiales más frecuentes en las excavaciones de época histórica: la cerámica. Aunque se trata de una muestra específica, confeccionada para el desarrollo de una Tesis Doctoral concreta (ESCRIBANO-RUIZ, 2014), es tan poco representativa y está tan fragmentada como cualquier otra. Por tanto, los problemas de fondo son universales para la cerámica arqueológica y las soluciones extrapolables a muchos otros contextos temporales y/o espaciales. Nos hemos animado a exponer este caso de forma pública y explícita porque, a pesar de percibir que la tradición de la cuantificación cerámica está cada vez más afianzada en los estudios de época post-clásica europeos, no existen trabajos escritos en castellano que aborden el problema de forma abierta y en todas sus facetas, desde el significado y los criterios de confección de la muestra, hasta la determinación taxonómica de los individuos cerámicos y la replicabilidad de los resultados. Queremos exponer nuestra estrategia cuantitativa, desarrollada, mejorada y validada a lo largo de nuestra experiencia doctoral; para llenar parcialmente ese vacío y contribuir, en nuestra medida, en el desarrollo y consolidación de las técnicas cuantitativas en arqueología en general, y en los estudios cerámicos en concreto.

\section{CONTEXTOS E INDIVIDUOS CERÁMICOS. ALGUNAS CONSIDERACIONES ESTADÍSTICAS Y ESTRATIGRÁFICAS PRELIMINARES}

Nuestro objetivo en la aludida Tesis Doctoral era el estudio de la cerámica alavesa de los siglos XIV al XVII pero es obviamente imposible afrontar el estudio de toda la población cerámica alavesa de los siglos aludidos, entendida bien como un conjunto homogéneo y bien definido que es objeto de estudio (GONZÁLEZ MANTEIGA, PÉREZ DE VARGAS, 2009: 12), bien como el conjunto de observaciones posibles del fenómeno aleatorio que se está estudiando (MURUZABAL, 2005). Por tanto, sólo hemos analizado e interpretado una muestra, una parte de esa población a partir de la cual estimaremos los valores del resto de la población. Pero no hemos elegido la cerámica de forma arbitraria, seleccionando aquellos tipos que nos resultaran especialmente interesantes por su función o por características estéticas. Nuestra selección para el muestreo, al contrario, ha respondido al contexto arqueológico en el que se han recuperado varios depósitos que cumplen con unos requisitos predeterminados. Hemos realizado, por tanto, un muestreo por conglomerados (GONZÁLEZ MANTEIGA, PÉREZ DE VARGAS, 2009: 16), en el que "las unidades de muestreo que se seleccionan aleatoriamente no son las unidades de la población, sino, generalmente, los sitios en los que buscar esas unidades" (COLL, GUIJARRO, 1998: 301), sobre los que se realiza después un muestreo aleatorio simple (MURUZABAL, 2005: 10).

Recordemos, dada la importancia que tienen estos conceptos en la estrategia de muestreo y en la configuración final de la muestra, que un depósito arqueológico es un tipo de unidad estratigráfica concreta y que la cerámica recuperada en su seno conforma un contexto cerámico. En su defensa nos basaremos en la definición que N. Terrenato (2001) hace sobre el contexto. En concreto, nos interesa aquella que entiende que un contexto es "un conjunto de restos hallados en las mismas unidades estratigráficas..., que se estudian en su conjunto y no como un grupo inconmensurable" y que presenta "información relacionada entre sí" (TERRENATO, 2001: 92). Utilizar el contexto como unidad de muestreo aumenta las posibilidades interpretativas (desde el nivel cronológico al simbólico), frente al estudio de los objetos descontextualizados.

Coincidimos también con el citado autor cuando asegura que los contextos casi siempre nos llegan incompletos y que, salvo en contadas ocasiones, representan una fracción de proporciones desconocidas respecto a la totalidad (TERRENATO, 2001: 92). Por tanto, es necesario tanto valorar el grado de coherencia de los contextos cerámicos, atendiendo a la residualidad y a los índices de fragmentación, como ser cautos respecto a su capacidad de representación. Algunos autores defienden que una muestra arqueológica difícilmente puede ser valorada en cuanto a su representatividad, sobre todo porque el tránsito del contexto sistémico al contex- 
to arqueológico representa un proceso de transformación severo y aleatorio (ARCELIN, TUFFREAU-LIBRE, 1998). Por ello, se considera inútil, incluso peligroso, fijar arbitrariamente un índice de confianza que, por otra parte, es muy difícil de establecer matemáticamente. Se defiende que no es útil pretender establecer a priori un umbral de fiabilidad matemática para el muestreo y que debe retenerse una única obligación: definir claramente el método de selección de las UU.EE. utilizadas y las zonas topográficas analizadas en el yacimiento (ARCELIN, TUFFREAU-LIBRE, 1998: V).

\section{LA NECESIDAD DE UN PROTOCOLO SELECTI- VO DE LA MUESTRA ESTRICTO Y SISTEMÁTICO}

Aludíamos a la importancia de la selección de las UU.EE. de las que proceden los contextos cerámicos, en la medida en la que son las unidades de muestreo en las que identificar las unidades de población (o individuos cerámicos). En consecuencia, seleccionar y analizar de forma crítica los contextos cerámicos es un primer paso empírico esencial en la confección de la muestra, más si cabe en nuestro caso, en el que no existen centros de producción excavados y en el que la mayoría de conjuntos cerámicos estudiados proceden de unidades estratigráficas en los que la cerámica ha sido desechada tras su consumo. Nuestra muestra cerámica, la materia prima de nuestro relato sobre el pasado, ha sido creada tras un riguroso proceso de selección, que ha priorizado el estudio de los depósitos más fiables desde el punto de vista de los procesos de formación de la estratificación. Aunque también ha sido importante que los contextos cerámicos contasen con diferentes indicadores cronológicos, este factor ha pasado a un segundo plano y ha estado supeditado siempre a los criterios estratigráficos.

En nuestro caso de estudio, la idoneidad de los contextos cerámicos ha estado determinada por las siguientes características:

- Que procedan de excavaciones llevadas a cabo siguiendo el procedimiento de excavación estratigráfica y utilizando como elemento básico de análisis las Unidades Estratigráficas, que serán las que definan los contextos cerámicos. Entendemos éstas como porciones de materia relativamente homogénea e indivisible, por lo que los componentes como los materiales arqueológicos son equivalentes y su posición se convierte en intercambiable en el seno de aquellos (CARANDINI, 1997:72). Asumir esta circunstancia nos permitirá utilizar las UU.EE. como marco contextual de referencia, y analizar los objetos que contiene como una consecuencia de una misma y última acción. Como un conjunto equivalente aunque mediatizado por procesos de equifinalidad, que pueden suponer el mismo resultado a partir de estados iniciales diferentes (LYMAN, 2004; ESCRIBANO-RUIZ, 2011: 110). A large number of factors, many of which are linked, may contribute to the stratigraphic product, and likely more than anywhere else the notion 'equifinality' is applicable to stratigraphic successions (POSTMA, VAN DEN BERG, 2008).

- Que siendo estratos excavados y documentados siguiendo la estratificación, presenten también indicadores cronológicos; ya que el muestreo ha seguido, además de los condicionantes geográficos aludidos, unas evidentes directrices cronológicas. El objetivo es contar con depósitos que además de presentar cerámica relevante, estén fechados de la mejor forma posible, bien sea mediante radiocarbono, monedas, datos procedentes de la documentación escrita, evidencias arquitectónicas y epigráficas, importaciones cerámicas conocidas o bien mediante todas o varias de ellas. Sin que una evidencia prevalezca por defecto sobre la otra, potenciando más su condición contextual que su consideración circunstancial de fósil guía. No obstante, estos elementos no tienen porqué encontrarse en el mismo depósito, ya que en muchas ocasiones la cronología de los estratos depositados por encima y por debajo del contexto en cuestión es suficiente para fijar una fecha precisa, con lo que la secuencia estratigráfica se convierte en un elemento de datación absoluta (SOLAUN, 2005:83). Ante todo, deberán estar contextualizadas, como decíamos, en una secuencia que marque las pautas de estratificación en las que ese determinado contexto se halla inmerso y cuya posición estratigráfica será en sí misma una referencia cronológica contextual primaria o secundaria.

- Que siendo estratos excavados y documentados siguiendo la estratificación, y presentando también indicadores cronológicos fiables, cuenten con un corpus cerámico mínimo. El requisito que finalmente hemos establecido para que los contextos pasen a formar parte de la muestra, es que al menos cuenten con más de 5 individuos computados mediante un protocolo de cuantificación que aúna las técnicas del Número mínimo de Individuos (Nml) y el Evaluated Vessel Equivalent (EVE), en la que ahondaremos más adelante.

- Que, además de todo lo anterior, cuenten con unas garantías mínimas respecto a los efectos transformadores generados por los procesos de formación del registro arqueológico. Por un lado, atendiendo a la presencia de material residual, aquel producido en una época precedente a aquella en la que se produce su ingreso en la estratificación arqueológica (TERRENATO, RICCI, 1998: 89) y descartando sistemáticamente los contextos que lo contienen de la muestra de referencia. Algunos autores (ARCELIN, TUFFREAU-LIBRE, 1998: XII) nos advierten de las implicaciones de omitir los elementos residuales y otros destacan su potencial interpretativo (TERRENATO, RICCI, 1998: 90). Nosotros creemos que la consideración de la residualidad es especialmente útil a la hora de entender los procesos de formación de la estratificación y guiarnos en la selección de los contextos, pero no para acercarnos al contexto sistémico de la cerámica. Por ello hemos decidido excluir de nuestro estudio aquellos conjuntos que presentaban cerámica residual muy clara y distante 
en el tiempo, como la terra sigillata. Su presencia, no muy frecuente y relacionada con acciones que inciden en la estratificación inferior, no nos ha proporcionado la confianza estratigráfica suficiente como para incorporar esos contextos en la muestra de referencia (de vocación informativa cuantitativa y cualitativa), por lo que han sido consecuentemente utilizados para proporcionar información exclusivamente cualitativa. Por otro lado, prestando atención a la dinámica de fragmentación de la cerámica en su contexto estratigráfico, análisis que expresado de forma numérica permite establecer índices de fragmentación que, a su vez, inspiraran mayor o menor confianza (ESCRIBANO-RUIZ, 2011). Finalmente, atendiendo a las pautas cronológicas de todos los componentes materiales de los contextos estratigráficos; cuya heterogeneidad ha sido considerado un indicador negativo, que puede acarrear la discriminación del conjunto cerámico, mientras que su homogeneidad ha sido concebido como un garante respecto a los efectos de los procesos de formación de la estratificación.

Los conjuntos cerámicos procedentes de Unidades Estratigráficas que han cumplido con los requisitos enumerados, han conformado la muestra de referencia; los que no han cumplido con dichas exigencias también han sido objeto de análisis en este trabajo, en condición de contextos informativos. Hemos seguido la premisa que dicta que una cantidad menor de datos de buena calidad es preferible a una cantidad mayor de información de baja calidad (ORTON, 2000). Aunque la interpretación final se ha alimentado de los datos procedentes de ambos tipos de contextos, consecuentemente su rol no ha sido en absoluto el mismo en el relato final. Como ya hemos adelantado, la muestra ha sido utilizada para realizar la mayor parte de las consideraciones cuantitativas y cualitativas, y ha conformado la base y estructura argumental. Por su parte, los contextos informativos únicamente han contribuido con información cualitativa de forma puntual, aportando al discurso una argumentación eventual siempre contextualizada en el discurso argumentativo derivado de la muestra referencial.

\section{DETERMINAR LA REPRESENTATIVIDAD CE- RÁMICA DEL REGISTRO ARQUEOLÓGICO $O$ CUANTIFICAR}

\subsection{Breve síntesis historiográfica de las técnicas cuantitativas aplicadas a la cerámica}

Tras analizar las características de los contextos estratigráficos y definir los conjuntos cerámicos que conformarán la muestra sobre la que elaboraremos nuestras conclusiones, el siguiente paso empírico es determinar cuantitativamente su diversidad, definir "su población" o cuantificar. Es el momento de precisar cómo hemos definido las unidades de la población, los individuos cerámicos que se encuentran en cada unidad de muestreo.

La literatura sobre cuantificación arqueológica en general, y sobre cerámica en particular, no es muy extensa, y se encuentra condicionada, además, por tradiciones colectivas. Por ello, las técnicas utilizadas están más vinculadas a los arqueólogos que a la realidad de los datos (HUSI, 2001: 4). Estas circunstancias suponen que las síntesis sobre cuantificación ofrezcan una visión parcial que, en ocasiones, no converge ni en los aspectos más básicos. Basta con comparar las síntesis recogidas en tres manuales editados en diferentes países, publicados en un margen cronológico de 14 años para confirmar esta rotunda afirmación (RICE, 1987: 291-292; ORTON, TYERS, VINCE, 1997: 188-206, MOLINARI, 2001: 56). Por eso es de agradecer la existencia de iniciativas que han generado modelos de cuantificación específicos, como el ya legendario Protocolo de Beuvray (ARCELIN, TUFFREAU-LIBRE, 1998) o el más reciente Protocolo de Sevilla (ADROHER AUROUX et al., 2016), que -aún estando circunscritos a un país o periodo concreto- pretenden unificar procedimientos analíticos y hacer comparables los resultados a una escala mayor que la tradicional, ceñida a grupos de trabajo específicos y yacimientos concretos.

A pesar todas las técnicas de cuantificación pretenden lo mismo, su planteamiento y diseño conceptual varían sobremanera de una a otra técnica, y la imagen general que se obtiene de cualquier intento de síntesis es la de una historiografía que por lo general se ha desarrollado en paralelo de forma voluntaria. Esto puede suceder tanto dentro de una extensión geográfica determinada, como a nivel internacional. En algunos países existen claras preferencias que marcan tendencia a la hora de cuantificar la cerámica histórica, como el EVE en el Reino Unido (ORTON, TYERS, VINCE, 1997: 188-204) y el Nml en Francia (ARCELIN, TUFFREAU-LIBRE, 1998) o en los Estados Unidos (VOSS, ALLEN, 2010). En otros, como en el caso de los Países Bajos, coexisten distintas tradiciones cuantitativas sin que exista una aparentemente clara preeminencia (POULAIN, 2013: 108-110). En esta última casuística se enmarca también el caso del estado español, donde sólo recientemente se ha comenzado a explicitar las técnicas cuantitativas empleadas. El balance historiográfico dibuja un panorama dividido, que escapa del tradicional esquema de las dos técnicas estimativas principales. Por un lado, están los autores más influenciados por la historiografía francesa y la arqueología clásica, que cuantifican mediante NmI (URTEAGA, AMUNDARAY, 2003; SOLAUN, 2005; QUEVEDO, 2013). Por otro lado se ha consolidado una escuela de sede meridional que, siguiendo la historiografía británica, cuantifica mediante la técnica del EVE (JIMÉNEZ PUERTAS 2012, ABELLEIRA DURAN, 2014, BUSTO ZAPICO, 2015; MARTÍNEZ ALVAREZ, 2015). Existen, asimismo, otras propuestas más minoritarias que optan por el cálculo del Número Máximo de Individuos, nMi (AMORÓS, 2013; BUXEDA i GARRIGÓS, MADRID i FERNÁNDEZ, 2008, 2016).

Pero la realidad se resiste, como de costumbre, a esquemas analíticos unilineales y se reconoce la necesidad de utilizar de forma conjunta diferentes técnicas (HUSI, 2001: 4; MOLINARI, 2001: 56; ORTON, TYERS, VINCE, 1997: 36; ADROHER AUROUX, SÁNCHEZ MORENO, DE 
LA TORRE CASTELLANO, 2015, 2016; ADROHER AUROUX et al., 2016). En palabras de estos autores, el diseño analítico cuantitativo debería ajustarse tanto al problema que se pretenda encarar, como a la naturaleza de los datos disponibles y de la muestra. Trataremos de explicar a continuación nuestra propuesta, que se encuadra en esta proclama pragmática.

\subsection{Un ejemplo de sistema de cuantificación dual}

Aunque en nuestros trabajos iniciales (ESCRIBANO-RUIZ, 2006; SOLAUN, ESCRIBANO-RUIZ, 2006) defendimos el empleo exclusivo de las técnicas de estimación de vasijas, al proponer el empleo simultáneo del EVE (Evaluated Vessel Equivalent) y el NmI (número mínimo de individuos); posteriormente interiorizamos que cada técnica puede y debe ser utilizada para diferentes fines, e incorporamos a nuestro acervo cuantitativo los cómputos de frecuencia de fragmentos (ESCRIBANO-RUIZ, 2011, 2014). El uso conjunto de ambas aproximaciones cuantitativas en nuestra investigación, desde el mismo proceso selectivo de la muestra, nos ha proporcionado una confianza mayor en nuestras interpretaciones y si nos preguntaran si podemos confiar en nuestra diversidad (BUXEDA i GARRIGÓS, MADRID i FERNÁNDEZ, 2008), estaríamos más seguros de nuestra respuesta afirmativa.

Tal y como cabe esperar de una muestra cerámica procedente de intervenciones arqueológicas, las piezas completas son muy raras en nuestra muestra, con mucha diferencia las menos representadas. Por ello, los principales problemas a los que nos enfrentamos son la fragmentación a la que ha sido sometida la muestra cerámica, y la integridad del contexto cerámico arqueológico respecto a su contexto sistémico, aspectos ambos mediados por los procesos de formación del registro arqueológico. Pero creemos que esta circunstancia problemática, que genera serias dificultades tanto a la hora de caracterizar la población cerámica como a la hora de comprenderla, puede ser mitigada con el empleo de diferentes técnicas de cuantificación. Por un lado, los problemas de inferencia producidos por la fragmentación a la hora de interpretar el contexto sistémico de la cerámica, pueden ser aminorados mediante el empleo de técnicas de estimación de vasijas, anteponiendo preferentemente las características composicionales de la cerámica a su forma. Por otro lado, como ya hemos apuntado, la coherencia del conjunto cerámico, que determinará la selección de la muestra, puede ser valorada mediante el uso de índices derivados de la cuantificación de frecuencia de fragmentos.

En otras palabras, los problemas que los procesos de formación generan en la muestra en forma de fragmentación y pautas de deposición aleatorias, pueden ser encarados mediante las técnicas de cuantificación de dos maneras diferentes y complementarias: a) De forma estratigráfica: aprovechando la fragmentación para valorar la coherencia de los conjuntos cerámicos, y seleccionar consecuentemente los contextos que compondrán la muestra cerámica de referencia; siendo determinante, a este respecto, el índice de fragmentación de cada contexto. b). De forma taxonómica: utilizando técnicas que obvien la fragmentación a la hora de establecer la cantidad de vasijas representadas por los fragmentos y obtengan valores cuantitativos más realistas; pretendido, en nuestro caso, mediante el empleo simultáneo del EVEy el Nml.

a) La valoración estratigráfica de los contextos cerámicos (nMi, NR, IF)

Tal y como anunciábamos, la fragmentación puede ayudarnos a comprender la dinámica de los procesos de formación de la estratificación de un yacimiento y a valorar la integridad de los diferentes depósitos arqueológicos de cara a la selección del material cerámico digno de estudio. De este modo, se propone el uso de determinadas técnicas que evalúen cuantitativamente la fragmentación de cada depósito, para poder valorar, a su vez, la integridad y coherencia interna de los contextos cerámicos. Se presupone que seleccionar los contextos arqueológicos menos afectados por las acciones posteriores a su deposición, ofrece una garantía interpretativa en nuestro intento de acercarnos al contexto sistémico de la cerámica, sus pautas de producción y consumo en determinados momentos históricos.

Para mensurar la fragmentación de cada contexto hemos calculado su índice de fragmentación o IF (sensu TUSET, 1991; BUXEDA i GARRIGÓS, MADRID I FERNÁNDEZ, 2008, 2016: 33), obtenido mediante la división del Número Máximo de Individuos $(n M i)$ y del Número de Restos (NR): IF = nMi / NR (ESCRIBANO RUIZ, 2011: 113). Se trata de valorar numéricamente el grado de relación de la cerámica recuperada en un mismo contexto, determinar si el conjunto cerámico depositado mediante una misma acción estratigráfica, pero recuperado en una ubicación espacial distinta, tienen relación entre sí, o si cada fragmento representa un caso aislado. Hipotéticamente, cuanto mayor sea el grado de relación mayor será la confianza en el contexto. Por tanto, aunque deberíamos estudiar aquellos contextos que estuvieran más cerca del valor 0 , finalmente decidimos ser relativamente generosos y establecer el umbral en 0,90 (IF 0,90$)$, descartando todos los contextos que presenten un valor superior. Al fin y al cabo, este umbral permite afirmar que los materiales están mínimamente relacionados. Quizá, si hubiéramos abordado la tesis en el momento de su redacción final, hubiéramos sido más estrictos con este requisito; sin embargo, por optimizar el tiempo invertido en el análisis cerámico y por ser contextos muy representativos de la arqueología urbana, decidimos valorar también aquellos que cuentan con índices relativamente altos.

Aunque en nuestro caso hemos debido calcular los valores de las dos formas de cuantificación necesarias para establecer el IF (nMi y NR), estos valores 
podrían ser fáciles de añadir a los inventarios de cerámica. Dado que son técnicas de cuantificación de base descriptiva, proporcionan un diagnóstico cuantitativo detallado y exhaustivo del contenido de cada contexto estratigráfico y colaboran en el principal cometido de los inventarios; en otras palabras, determinar con precisión el número de fragmentos recuperados en cada contexto arqueológico. Su implementación en el procedimiento de inventariado sería relativamente sencilla. Cada número de inventario sería equivalente a cada individuo cerámico cuantificado mediante Número Máximo de Individuos ( $n M i)$ y en la descripción de cada individuo se recogería sistemáticamente el número de fragmentos por los que está compuesto; es decir, el número de restos (NR) que lo componen. Este simple procedimiento permitiría contar con inventarios en cuya elaboración se cuantificara de forma consciente y ofrecer datos que permitieran establecer el índice de fragmentación de cada contexto de forma inmediata. Es, además, un modelo claro y su empleo no se traduce en una pérdida de información descriptiva como sucede, a menudo, con los inventarios que se ajustan al Número Mínimo de Individuos ( $\mathrm{Nm} /$ ).

b) La determinación taxonomíca de la población cerámica $(N m l, E V E)$

Si hemos justificado la elección del Índice de Fragmentación (IF = nMi / NR) como medida de valoración del contexto arqueológico, es necesario exponer ahora los motivos que nos han inducido a utilizar el binomio Nml-EVE para intentar acercarnos al contexto sistémico de la cerámica, a los individuos que forman parte de la muestra y a partir del cual explicaremos el resto de la población. Finalmente, también expondremos el modo en el que lo hemos calculado su valor.

La elección exclusiva y empleo sistemático del Nml-EVE como herramienta para crear nuestra base interpretativa, responde a diferentes razones. En primer lugar, porque en nuestro caso el objetivo principal de la cuantificación, no era tanto determinar el tamaño total de cada conjunto, sino establecer las proporciones de los distintos tipos que lo constituyen (ORTON, 1993: 169; ORTON, TYERS, VINCE, 1997: 188). Y precisamente el potencial del $\mathrm{Nml}$ "reside en la estimación de la relación entre las distintas partes" (CHAIX, MÉNIEL; 2005: 167); es decir, que a diferencia de otras técnicas, nos permite comparar las proporciones de los tipos pertenecientes a los distintos conjuntos (ORTON, TYERS, VINCE, 1997: 194). Por tanto, cuantificar siguiendo el sistema señalado nos permitirá valorar y representar de la forma más eficaz posible la diversidad cerámica del conjunto de UU.EE. que forman la muestra. A su vez, como las variaciones de los tipos o de las producciones cerámicas son los mejores indicadores cronológicos (MOLINARI, 2001: 56; HUSI, 2001: 8, 13), cuantificar siguiendo el sistema propuesto también nos permitirá crear una herramienta que permita utilizar dichas dataciones a nivel local, y en algunas ocasiones, a nivel regional e incluso supraregional.
En segundo lugar, la elección de este sistema también nos permite evitar el sesgo producido por la fragmentación en los contextos que, como hemos podido comprobar, condiciona a las técnicas basadas en la frecuencia de fragmentos (GAIMSTER, 2006: 48; BYRD, OWENS, 1997: 315-316). El problema de la sobrestimación que genera la fragmentación en los cómputos mediante NR y nMi (RICE, 1987: 291), nos parece suficientemente importante como para no utilizar dichas técnicas en la consideración del contexto sistémico de la cerámica. Creemos que este mecanismo es esencial por nuestra forma de proceder, adoptada en ausencia de otras posibilidades y desarrollada por considerar que es la vía de acercamiento más eficaz. Si para acercarnos lo máximo posible a ese live assemblage, o contexto sistémico de la cerámica, tenemos que partir de la consideración del dead assemblage, o contexto arqueológico, es inevitable tratar de mitigar los efectos transformadores creados por las pautas de desecho y por los posteriores procesos de formación y transformación del registro arqueológico. Y entre ellos, uno de los más importantes es, sin duda, la fragmentación. En algunos casos, como el ilustrado en la Figura 1, estamos muy seguros que determinados fragmentos pertenecen a una misma pieza $(N m /-E V E=1)$, pero el Número Máximo de Individuos dice que son 4 y el Número de Restos determina que son 7.

En nuestro caso de estudio, sólo un contexto de los 32 que conforman el conjunto de contextos de referencia presentan un Índice de Fragmentación inferior a $0,5(I F \leq 0,50)$ y otros tantos han sido descartados de ese conjunto de contextos referenciales por presentar

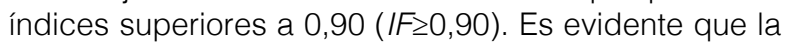
fragmentación condiciona seriamente nuestra muestra. Es necesario, por tanto, utilizar una técnica que mitigue su efecto en la interpretación del contexto sistémico de la cerámica y que nos permita acercarnos de forma más precisa al volumen de la cerámica consumido en determinado periodo, sobreestimado cuando se emplean técnicas condicionadas por la fragmentación, como el nMi o el NR. Si utilizáramos estas últimas técnicas, y no el $\mathrm{Nml}$, renunciaríamos a la convicción que nos proporciona la capacidad interpretativa que cualquier arqueólogo desarrolla al rastrear las posibles asociaciones entre las distintos fragmentos que componen un contexto. Son tantas las ocasiones en las que estamos seguros que varios fragmentos se asocian a la misma pieza, pero en los que no existe una relación de "pegado" que lo demuestre (como en el caso de la Figura 1); y tan significativas sus implicaciones en la interpretación cuantitativa y cualitativa de la cerámica, que no estamos dispuestos a renunciar a su empleo en este estudio.

Finalmente, podemos argumentar en su favor que el $\mathrm{Nm} /$ asegura la representación de los tipos cerámicos minoritarios (GAIMSTER, 2006: 48). Esta circunstancia también puede ser considerada como un efecto negativo, porque generalmente mediante esta técnica se 


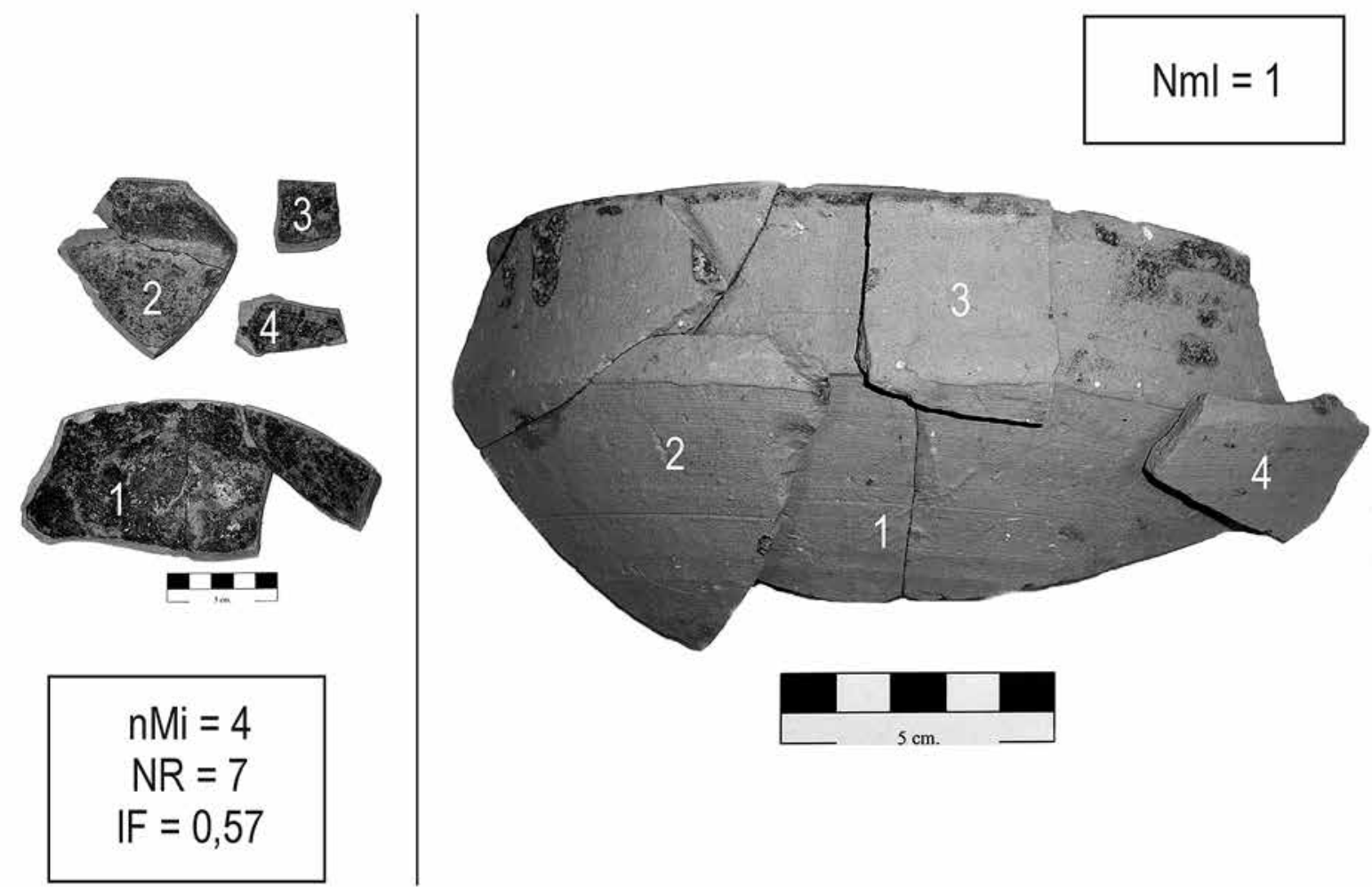

Fig. 1. Ejemplo del cálculo del Número mínimo de Individuos e ilustración de la evidente correspondencia de los fragmentos a una misma pieza (adaptado de Escribano-Ruiz, 2011: 116). / An example of estimation of Minimum Number of Individuals and illustration showing the correspondence of different fragments to the same bowl.

sobrevaloran las producciones menos comunes frente a las más representadas, cuya presencia tiende a ser infravalorada (HUSI, 2001: 7). Esto ha sido mitigado, en nuestro caso, incorporando al $\mathrm{Nm} /$ propuesto en el protocolo de Beuvray algunas de las consideraciones del $E V E$, como la determinación de la proporción de cada individuo; muy fácil de calcular si empleamos un gráfico de bordes porcentual ( $p$. ej. ORTON, TYERS, VINCE, 1997: 196). De esta manera, si conservamos un borde completo y medio más del mismo diámetro, no consideraremos que únicamente representa a una pieza (como haríamos si empleamos exclusivamente el cálculo del

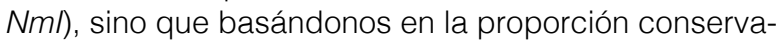
da estableceremos la presencia de dos piezas iguales. Como cabe suponer, el problema de la sobrevaloración de los tipos menos comunes, también es menos manifiesto cuando los tipos predominantes cuentan con más elementos de juicio que las pastas o los acabados superficiales simples (por ejemplo, vidriados de distinto color, una decoración pintada, o más incluso si es polícroma). Pero estas últimas circunstancias no han sido habituales en nuestro estudio, ya que la mayoría del registro cerámico responde al tipo de cerámica sin revestir y sin decorar.

Por tratar de evaluar hasta qué punto se infravaloran las producciones más habituales, que cuentan además con menos variables de análisis, hemos realizado una prueba empírica, cuyos resultados se presentan en la siguiente tabla (Tabla I). Por simplificar la comparación, los datos no se presentan por grupos cerámicos sino por tipos genéricos: vidriado (vidriado), vidriado blanco ( vidriado b) o sin vidriar. En este esquema los tipos vidriados encarnan el rol de supuestos productos supravalorados por el Nml-EVE y la cerámica sin vidriar asume el papel de productos infravalorados.

Las conclusiones que nos aporta la interpretación de los datos de la Tabla I, son muy reveladoras y demuestran que sí existe tal sobrerrepresentación, que efectivamente con el empleo del Nml-EVE se incrementa la presencia de los tipos menos presentes. La diferencia entre los porcentajes alcanzados mediante las dos técnicas ha sido muy significativa en algunos casos, muy cercana al $40 \%$ en el ejemplo más extremo, aunque en otros no lo ha sido tanto, en torno al $7 \%$ en el caso más cercano. La diferencia media es de un $18 \%$, en el caso de los tipos sin vidriar (Nml-EVE 68,5\% // nMi $86,5 \%$ ), de un $14 \%$ en el caso de los tipos con vidriado (Nml-EVE 25,5\% // nMi 11,5\%) y de un 4\% en el caso de los tipos vidriados en blanco (Nml-EVE 6\% // nMi 2\%).

A pesar de estos resultados y siendo uno de los objetivos principales determinar la diversidad cerámica de los contextos, consideramos que es la técnica más positiva y que, como el resto, no está exenta de determinados problemas que es necesario tener en conside- 


\begin{tabular}{|c|c|c|c|c|c|c|}
\hline UE & NmI-EVE & nMi & & UE & NMI-EVE & NMI \\
\hline SMC.05.26582 & 8 & 37 & & SMC.05.26559 & 10 & 36 \\
\hline Sin vidriar & $75 \%$ & $92 \%$ & & Sin vidriar & $90 \%$ & $97,5 \%$ \\
\hline Vidriado & $25 \%$ & $8 \%$ & & Vidriado & $10 \%$ & $2,5 \%$ \\
\hline Vidriado b & $0 \%$ & $0 \%$ & & Vidriado b & $0 \%$ & $0 \%$ \\
\hline & & & & & & \\
\hline SMC.05.26770 & 23 & 123 & & SMC.05.27445 & 8 & 32 \\
\hline Sin vidriar & $91,5 \%$ & $98,5 \%$ & & Sin vidriar & $87,5 \%$ & $97 \%$ \\
\hline Vidriado & $8,5 \%$ & $1,5 \%$ & & Vidriado & $12,5 \%$ & $3 \%$ \\
\hline Vidriado b & $0 \%$ & $0 \%$ & & Vidriado b & $0 \%$ & $0 \%$ \\
\hline
\end{tabular}

\begin{tabular}{|c|c|c|c|c|c|c|}
\hline UE & NmI-EVE & nMi & & UE & NMI-EVE & NMI \\
\hline SMC.05.26609 & 10 & 33 & & SMC.05.26174 & 9 & 74 \\
\hline Sin vidriar & $80 \%$ & $94 \%$ & & Sin vidriar & $55,5 \%$ & $94 \%$ \\
\hline Vidriado & $10 \%$ & $3 \%$ & & Vidriado & $45,5 \%$ & $0 \%$ \\
\hline Vidriado b & $10 \%$ & $3 \%$ & & Vidriado b & $0 \%$ & $0 \%$ \\
\hline COC.02.2005 & 21 & 42 & & VIT.XIII.03.438 & 29 & 69 \\
\hline Sin vidriar & $43 \%$ & $69 \%$ & & Sin vidriar & $27,5 \%$ & $55,5 \%$ \\
\hline Vidriado & $57 \%$ & $41 \%$ & & Vidriado & $38 \%$ & $29 \%$ \\
\hline Vidriado b & $0 \%$ & $0 \%$ & & Vidriado b & $34,5 \%$ & $15,5 \%$ \\
\hline
\end{tabular}

Tabla 1: Resultados de los tipos generales de cerámica, con distintas técnicas aplicadas a los mismos contextos. / Results for the general types of pottery, with different techniques applied to the same context.

ración. $Y$, en nuestra opinión, el uso del Nml-EVE para acercarnos al contexto sistémico de la cerámica, tiene más ventajas que problemas: además de asegurar la representación de los tipos minoritarios, es adecuado para establecer las proporciones de los distintos tipos de cerámica y, sobre todo, es capaz de aproximarse cuantitativamente a la población cerámica de forma más precisa por no atender a la fragmentación. Porque la gente no usaba fragmentos sino vasijas, esta técnica nos acerca más a la comprensión de los objetos utilizados por las comunidades históricas que estudiamos (VOSS, ALLEN, 2010: 1, 9).

En cuanto a su ejecución, el protocolo seguido en nuestro caso puede resumirse de la siguiente manera. Una vez realizado el análisis macroscópico de todos y cada uno de los fragmentos de cada Unidad Estratigráfica y establecidas las agrupaciones tecnotipológicas preliminares (sensu ESCRIBANO-RUIZ, 2012: 232233), se clasifican las formas y se valora la cantidad de vasijas que representan. Los requisitos básicos de este sistema combinado son:

- que la cuantificación haya sido realizada por Unidades Estratigráficas

- que todos los tipos cerámicos hayan sido definidos a partir de su pasta y todos fragmentos consecuentemente adscritos a un grupo cerámico determinado

- que sean cuantificadas preferentemente las partes de las vasijas que podamos atribuir a una forma específica (principalmente el borde, aunque también las asas o la decoración), o en su defecto las que representen a un grupo cerámico del que no se conserve algunas de las partes mencionadas.

\section{REPLICABILIDAD Y EXTRAPOLACIÓN DE LOS RESULTADOS}

Inevitablemente nos hemos preguntado si los resultados cuantitativos alcanzados en nuestro estudio son lo suficientemente sólidos y significativos como para ser utilizados de referencia en otras investigaciones. Durante nuestra experiencia hemos podido comprobar que la diversidad de tipos que convergen en los contextos es un instrumento de datación, especialmente a nivel local. Por ello hemos tratado de asegurar la coherencia interna de los contextos seleccionados, y hemos sido muy determinantes al respecto. Ahora pretendemos comprobar si las proporciones de distintos tipos de cerámica se pueden replicar y, por tanto, si este trabajo crea indicadores cronológicos que pueden ser extrapolables.

La pregunta que explica este apartado y dirige nuestra argumentación durante su desarrollo es: ¿Son significativos, y por tanto extrapolables, los resultados alcanzados en nuestro estudio? Nuestra premisa de partida es que una investigación que cuenta con una muestra compuesta por cientos de miles de fragmentos, proporciona una información de naturaleza estruc- 
tural, en la que se consideran todas las excepciones y que por ello resulta ajena a los episodios eventuales que puede representar un contexto concreto. Pero de esta cuestión se derivan otras preguntas de naturaleza metodológica: ¿es fiable la proporción de tipos establecida a partir de la suma de individuos Nml-EVE?, ¿si realizamos un estudio con otros contextos distintos, los porcentajes serían similares?

Gracias a las distintas etapas, y trabajos puntuales que jalonan el extenso desarrollo de nuestro trabajo, podemos ofrecer una respuesta concreta, y afirmativa, a estas preguntas. La comparación de los porcentajes de cerámica vidriada ${ }^{1}$ y sin vidriar de un yacimiento, la Catedral Santa María de Vitoria-Gasteiz, en un siglo concreto, el XIV, nos permite testar la respuesta del registro cerámico a nuestro sistema de cuantificación. Estos porcentajes provienen de tres trabajos distintos, la tesis doctoral de la que se deriva gran parte de este texto (ESCRIBANO-RUIZ, 2014), el trabajo presentado en el décimo Congreso Internacional de la Cerámica Medieval en el Mediterráneo (ESCRIBANO-RUIZ, SOLAUN, 2016) y el Trabajo de Investigación presentado para obtener el Diploma de Estudios Avanzados (ESCRIBANO-RUIZ, 2006). Tal y como puede ser observado en la tabla que se muestra a continuación (Tabla II), tres estudios realizados usando diferentes UU.EE. ${ }^{2}$, aportan proporciones muy similares y presentan una desviación máxima del 3\%. Por ello consideramos que los resultados son replicables, que el procedimiento seguido es fiable y que los resultados son, en consecuencia, representativos.

\begin{tabular}{|c|c|c|c|}
\hline & Muestra Tesis & $\mathbf{X}$ CICM (Silves) & Trabajo DEA \\
\hline NMI-EVE & 126 & 207 & 100 \\
\hline sin vidriar & $90 \%$ & $90 \%$ & $93 \%$ \\
\hline vidriado & $8 \%$ & $9 \%$ & $6 \%$ \\
\hline vidriado B. & $2 \%$ & $1 \%$ & $1 \%$ \\
\hline
\end{tabular}

Tabla 2: Porcentajes de los distintos tipos cerámicos utilizando contextos diferentes del siglo XIV. / Percentages of the different pottery types using diverse contexts dated in the $14^{\text {th }}$ century.

Hemos podido comprobar que, en la práctica, cuando el corpus cerámico es amplio, nos aseguramos la existencia de una información de base estructural homogénea, desprovista de una gran aleatoriedad. Sin embargo, aún debemos valorar si esta información puede ser extrapolada y aplicada en la datación de contextos cerámicos individuales. Si bien creemos que no existe problema cuando se trata de contextos con una cantidad de cerámica abundante, los problemas son mayores a la hora de medir la diversidad de conjuntos cerámicos pequeños, para los que el empleo del Nml-EVE no siempre es tan adecuado. Por ello, a continuación planteamos algunos procedimientos alternativos y complementarios para comparar nuestros resultados, derivados de una estructura que procede de la suma de decenas de contextos, con contextos individuales o con estudios de distinta naturaleza.

En muchas ocasiones sucede que contamos con un único fragmento de un tipo cerámico y 10 de otro, y que en la estimación de individuos computa como 1 - 1. En estos casos, que exceden el ámbito del presente trabajo y son más cercanos al estudio cronológico de cada una de las UU.EE. de un yacimiento, es recomendable usar también el nMi como técnica para establecer una media con la que determinar la proporción de los distintos tipos de cerámica. Esta propuesta ha sido ratificada en las conversaciones mantenidas con J. L. Solaun, quien también ha comprobado que este procedimiento socava en parte los efectos negativos del $\mathrm{Nm}$ l en contextos de pequeñas dimensiones (SOLAUN, 2013: 212-213). Con la intención de comprobar la viabilidad de esta propuesta, hemos realizado otra prueba con algunos de los contextos del siglo XIV anteriores, cuyos valores y porcentajes pueden ser comparados con los obtenidos a partir de la muestra de referencia para el caso del mismo yacimiento, Catedral de Santa María de Vitoria-Gasteiz, y el mismo siglo, el XIV (Tabla III)".

Considerando ambos valores, y haciendo una media entre el Nml-EVEy el $n M i$, las proporciones establecidas para las UU.EE. con poca cerámica se asemejan más a las determinadas por la estructura que nos provee el estudio de los 126 individuos, y no se desvían de la tendencia principal en más de un 8,5\%. De forma significativa, las mayores diferencias se producen con los contextos que tienen el menor número de individuos Nml-EVE. Por ello planteamos la posibilidad de realizar esta aproximación mixta en aquellos contextos cerámicos compuestos por pocos fragmentos cuyos valores Nml-EVE no se ajustan a la diversidad que expresa el nMi o el NR. Al contrario, consideramos que en los contextos que cuenten con una población cerámica nutrida, no es necesario realizar esta aproximación alternativa para poder comparar los porcentajes con los de este trabajo, sino que lo aconsejable es comparar sus porcentajes calculados mediante NmI-EVE.

\footnotetext{
1 Dentro de la que se distingue el vidriado de plomo (vidriado) de aquel generalmente, no siempre, compuesto también por estaño y que presenta ante el consumidor como un vidriado de color blanco (vidriado B.). Para una justificación de esta opción véase ESCRIBANO-RUIZ, 2013: 55 .

2 Cada uno de los trabajos ha valorado entre 10 y 19 contextos y entre los tres trabajos sólo coinciden dos contextos. Entre el trabajo para el DEA y el presentado en Silves, coincide el $26 \%$ de los contextos y entre éste último y el trabajo actual, el $40 \%$. Las razones por las que variaron los contextos en cada trabajo son múltiples y variadas, y no vamos a detenernos en justificarlas.
} 


\begin{tabular}{|c|c|c|c|}
\hline U.E. & $N m l-E V E$ & $n M i$ & media \\
\hline 26489 & 9 & 48 & \\
\hline sin vidriar & $77.5 \%$ & $93.5 \%$ & $85,5 \%$ \\
\hline vidriado & $22.5 \%$ & $6.5 \%$ & $14,5 \%$ \\
\hline vidriado $B$ & $0 \%$ & $0 \%$ & $0 \%$ \\
\hline 26559 & 10 & 36 & \\
\hline sin vidriar & $90 \%$ & $97.5 \%$ & $93,75 \%$ \\
\hline vidriado & $10 \%$ & $2.5 \%$ & $6,25 \%$ \\
\hline vidriado $\mathrm{B}$. & $0 \%$ & $0 \%$ & $0 \%$ \\
\hline 26582 & 8 & 37 & \\
\hline sin vidriar & $75 \%$ & $92 \%$ & $83,5 \%$ \\
\hline vidriado & $25 \%$ & $8 \%$ & $16,5 \%$ \\
\hline vidriado $\mathrm{B}$. & $0 \%$ & $0 \%$ & $0 \%$ \\
\hline 26609 & 10 & 33 & \\
\hline sin vidriar & $80 \%$ & $94 \%$ & $87 \%$ \\
\hline vidriado & $10 \%$ & $3 \%$ & $6,5 \%$ \\
\hline vidriado $\mathrm{B}$. & $10 \%$ & $3 \%$ & $6,5 \%$ \\
\hline 26770 & 23 & 123 & \\
\hline sin vidriar & $91.5 \%$ & $98.5 \%$ & $95 \%$ \\
\hline vidriado & $8.5 \%$ & $1.5 \%$ & $5 \%$ \\
\hline vidriado $\mathrm{B}$. & $0 \%$ & $0 \%$ & $0 \%$ \\
\hline SIGLO XIV & 126 & & \\
\hline sin vidriar & $90 \%$ & & \\
\hline vidriado & $8 \%$ & & \\
\hline vidriado $\mathrm{B}$. & $2 \%$ & & \\
\hline
\end{tabular}

Tabla 3: Valores de diferentes contextos del siglo XIV por Nml-EVE y nMi y su media. / Values of different contexts dated in the 14th century, both using Nml-EVE and nMi, plus the mode.

\section{CONCLUSIONES}

El principal objetivo de este trabajo era ofrecer un ejemplo razonado de una estrategia de cuantificación concreta, que abarca tanto su diseño estadístico conceptual como la confección de la muestra o las técnicas concretas empleadas. Hemos tratado de conceptualizar cómo las unidades de excavación se convierten en unidades estadísticas y hemos propuesto un protocolo selectivo de contextos para confeccionar una muestra cerámica libre de grandes distorsiones generadas por los procesos de formación del registro arqueológico. Asimismo hemos tratado de explicar y justificar qué técnicas de cuantificación hemos utilizado y en qué ha contribuido cada una de ellas. Finalmente, hemos argumentado que nuestro trabajo puede ser utilizado de forma comparativa en otros trabajos, con las cautelas y procedimientos señalados. Este último aspecto es de gran relevancia porque avala la viabilidad de utilizar nuestro trabajo doctoral en los estudios arqueológicos alaveses o en aquellos lugares en los que aparezca cerámica alavesa. Es el caso, por ejemplo, de la cerámica recuperada en los yacimientos vascos de Canadá (ESCRIBANO-RUIZ, BARREIRO, 2016) cuyo uso comparativo debe tener en cuenta que esta información procede de contextos arqueológicos calibrados a nivel estratigráfico y tratados estadísticamente de esta manera concreta.

Debemos matizar, sin embargo, que aún queda debate en cuanto a la idoneidad de una u otra técnica cuantitativa para determinar el número de cerámicas pretéritas. Sería importante que se publicaran trabajos en los que se discutiera y problematizara esta cuestión, que no debe darse por zanjada. En el caso del potencial valorativo estratigráfico de la cerámica son necesarios más estudios que avancen en el camino marcado, que aún cuenta con un corto recorrido. Creemos que este es uno de los temas en los que la aportación de los estudios cuantitativos y cualitativos de la cerámica puede ser notable en el futuro cercano.

\section{AGRADECIMIENTOS}

La base de este texto se deriva de la Tesis Doctoral realizada por quien suscribe bajo la dirección de Agustin Azkarate (UPV/EHU) a quien debemos la base de nuestro conocimiento y nuestra principal muestra de agradecimiento. Este texto debe mucho a Jaume Buxeda (UB), nuestro mayor crítico y, al mismo tiempo, aliado en el laberinto de la cuantificación. La redacción final de este trabajo ha sido posible gracias a la convocatoria de 2015 de ayudas para la Especialización de Personal Investigador del Vicerrectorado de Investigación de la UPV/EHU. Agradecemos asimismo los comentarios de los revisores y/o revisoras, que han servido para aclarar, completar y mejorar tanto la forma como el contenido del texto.

\section{BIBLIOGRAFIA}

Abelleira duran, M. 2014 Origen, utilidad y límites teóricos de la cuantificación cerámica. Un aporte a la Arqueología Social Latinoamericana. @rqueología y territorio 11, 153-169.

Adroher Auroux, A. M.; Carreras Monfort, C.; de Almeida, R.; Fernández Fernández, A.; Molina Vidal, J. \& Viegas, C. 2016 Registro para la cuantificación de cerámica arqueológica: estado de la cuestión y una nueva propuesta. Protocolo de SeviIla (prcs/14), Zephyrus LXXVIII, 87-110.

Adroher Auroux, A. M. A., Moreno, A. S., \& de la Torre Castellano, I. 2015 Cuantificación en cerámica, ¿ejercicio especulativo o ejercicio hipotético? Las cerámicas ibéricas y púnicas en la lliberri del siglo IV aC procedentes del depósito de la calle Zacatín (Granada), Archivo Español de Arqueología 88, 39-65.

Adroher Auroux, A. M. A., Moreno, A. S., \& de la Torre Castellano, I. 2016 Cerámica ática de barniz negro de lliberri (Granada, España). Análisis crono-estadístico de un contexto cerrado, Portugalia 37, 5-38. 
Amorós, V. 2013 Tendencias tecnológicas de la cerámica altomedieval del Tolmo de Minateda, Mansio 1, 7-25.

Arcelin, P. \& Tuffreau-Libre, M. 1998 La quantification des céramiques, conditions et protocole, Actes de la table ronde du Centre archéologique européen du Mont Beuvray (Glux-enGlenne, 7-9 avril 1998). Centre archéologique européen du Mont Beuvray. Glux-en-Glenne.

Azkarate, A. 1999 Necrópolis tardoantigua de Aldaieta (Nanclares de Gamboa, Alava). Diputación Foral de Álava. Vitoria-Gasteiz.

Bailey, G. 2007 Time perspectives, palimpsests and the archaeology of time. Journal of anthropological archaeology 26, 198-223.

Busto Zapico, M. 2015 Sobre el uso de herramientas informáticas para la cuantificación cerámica: el EVE 2.0. SÉMATA, Ciencias sociais e humanidades 27, 199-219.

Buxeda i Garrigós, J. \& Madrid i Fernández, M. 2008 Individuals, assemblages and sampling in archaeometric studies. Can you trust your diversity? 5th Hellenic Society of Archaeometry Conference. Athens.

Buxeda i Garrigós, J. \& Madrid i Fernández, M. 2016 Designing Rigorous Research: Integrating Science and Archaeology, en Hunt A. M. W. The Oxford Handbook of Archaeological Ceramic Analysis, 19-47. Oxford University Press. Oxford.

Byrd, J.E. \& Owens, D.D. 1997 A Method for Measuring Relative Abundance of Fragmented Archaeological Ceramics. Journal of Field Archaeology 24 (3), 315-320.

Carandini, A. 1997 Historias en la Tierra. Manual de excavación arqueológica. Crítica. Barcelona

Chaix, L. \& Méniel, P. 2005 Manual de Arqueozoología. Ariel. Barcelona.

Coll, S. \& Guijarro, M. 1998 Estadística aplicada a la historia y a las ciencias sociales. Ediciones Pirámide. Madrid.

Escribano-Ruiz, S. 2006 Aproximación al estudio de los contextos cerámicos del País Vasco, siglos XIV-XVII. Trabajo de Investigación (Programa de Doctorado "Medio Ambiente, Territorio y Cultura: perspectivas desde la Geografía, la Prehistoria y la Arqueología", EHU-UPV). Vitoria-Gasteiz. https://addi. ehu.es/handle/10810/11684

Escribano-Ruiz, S. 2011 La cerámica en los procesos de formación, percepción e interpretación del registro arqueológico. Sobre el tránsito del contexto arqueológico al sistémico. Krei $11,109-118$

Escribano-Ruiz, S. 2012 El registro cerámico del País Vasco, Araba y Bizkaia, siglos XIV al XVII. Retrospectiva heurística, en Castro Correa, A., Gómez Castro, D., González Germain, G. Starczewska, K., Oller Guzmán, J., Puy Maeso, A., Riera Vargas, R. \& Villagra Hidalgo, N. Estudiar el pasado: Aspectos metodológicos de la investigación en Ciencias de la Antigüedad y de la Edad Media. 231-236. British Archaeological Reports International Series 2412. Oxford.

Escribano-Ruiz, S. 2013 Los antecedentes de la cerámica popular vasca. Consideraciones desde el consumo cerámico de Durango y Vitoria-Gasteiz (siglos XIV y XVII), en Gómez de Segura, B. Siglos de Alfarería en Ollerías. 34-61. Ayuntamiento de Legutio - Museo de Alfarería Vasca. Legutio.

Escribano-Ruiz, S. 2014 Genealogía del registro cerámico alavés de época preindustrial (siglos XIV al XVII). Tesis Doctoral inédita. Departamento de Geografía, Prehistoria y Arqueología, UPV/EHU. Vitoria-Gasteiz.

Escribano-Ruiz, S. 2016 Cerámica y comunidades de poder. La transvaloración del registro cerámico alavés entre el siglo
XIV y el XVII, en Ferri, M., Moine, C. \& Sabbionesi, L. In\&Around. Ceramiche e comunità. 219-227. All'Insegna Del Giglio. Firenze.

Escribano-Ruiz, S. \& Barreiro Argüelles, S. 2016 Traveling Ceramics: Basque Networks and Identities in the Gulf of Saint Lawrence, en Loewen, B. \& Chapdelaine, C. Contact in the 16th Century: Networks Among Fishers, Foragers and Farmers. 31-56. University of Ottawa Press. Ottawa.

Escribano-Ruiz, S. \& Solaun, J. L. 2016 La introducción de la cerámica vidriada en el Cantábrico Oriental (siglos XII-XV), en Gonçalves, M. J. \& Gómez Martínez, S. Actas do X Congresso Internacional a Cerâmica Medieval No Mediterrâneo. 534-548. Câmara Municipal de Silves \& Campo Arqueológico de Mértola. Silves.

Fernández Martínez, V. M. 2015 Arqueo-Estadística. Métodos cuantitativos en Arqueología. Alianza Editorial. Madrid.

Gaimster, D. R. M. 2006 The historical archaeology of pottery supply and demand in the Lower Rhineland, AD 1400-1800. Archaeopress. Oxford.

González Manteiga, M. T. \& Pérez de Vargas, A. 2009 Estadística aplicada. Una visión instrumental. Ediciones Diaz de Santos. Madrid.

Husi, P. 2001 Quantification et datation en céramologie (Le nombre minimum d'individus: la technique de quantification la mieux adaptée à la datation des contextes archéologiques à partir de l'exemple de Tours). Les petits cahiers d'Anatole 6 , $1-27$

Jiménez Puertas, M. 2012 El análisis cuantitativo de la cerámica medieval y los procesos de formación del registro arqueológico: estudio de un caso procedente del yacimiento de Madīnat Ilbīra. Debates de Arqueología Medieval 2, 293-329.

Lyman, R.L. 2004 The Concept of Equifinality in Taphonomy. Journal of Taphonomy 2 (1), 15-26.

Martinez Alvarez, C., 2015 Estrategia de muestreo cerámico para la periodización de los contextos arquelógicos localizados en la necrópolis medieval de Madinnat Ilbîra. Debates de Arqueología Medieval 5, 91-110.

Molinari, A. 2001 Cerámica, en Francovich, R. \& Manacorda D. Diccionario de Arqueología. 53-61. Crítica. Barcelona.

Muruzabal, J. J. 2005 Elementos de estadística. Teoría de muestras e inferencia estadística. Colegio de Ingenieros de caminos, canales y puertos. Madrid.

Olsen, B., Shanks ,M., Webmoor, T. \& Witmore, C. 2012 Archaeology. The Discipline of Things. University of California Press. Berkeley.

Orton, C. 1993 How many pots make five? An historical review of pottery quantification, Archaeometry 35 (2), 169-184.

Orton, C. 2000 Sampling in Archaeology. Cambridge University Press. Cambridge.

Orton, C., Tyers, P. \& Vince, A. 1997 La cerámica en Arqueología. Crítica. Barcelona.

Postma, G. \& Van den Berg, A.P. 2008 Impact of discharge, sediment flux and sea-level change on the stratigraphic architecture of river-delta-shelf systems, en de Boer, P., Postma, G., van der Zwan, C.J., Burgess, P. \& Kukla, P. Analogue and Numerical Modelling of Sedimentary Systems: From Understanding to Prediction. 191-205. Wiley-Blackwell. Oxford.

Poulain, M. 2013 Notes on the quantification of post-medieval pottery in the Low Countries. Post-Medieval Archaeology 47 (1), 106-118 
Quevedo, A. 2013 Contextos cerámicos y transformaciones urbanas en Carthago Noua: de Marco Aurelio a Diocleciano, Tesis Doctoral, Universidad de Murcia. www.tdx.cat/bitstream/10803/116883/1/TAQS.pdf

Rice, P.M. 1987 Pottery analysis: a sourcebook. The University of Chicago Press. Chicago.

Schiffer, M. B. 1987 Formation Processes of the Archaeological Record. University of New Mexico Press. Albuquerque

Solaun J. L. 2005 La cerámica medieval en el País Vasco (siglos VIII-XIII). Servicio Editorial de la Universidad del País Vasco, Gobierno Vasco. Bilbao.

Solaun J. L. 2013 La cerámica medieval en Gasteiz, en Azkarate, A. \& Solaun, J. L. Arqueología e Historia de una ciudad: Ios orígenes de Vitoria-Gasteiz. 195-244. Tomo I. Servicio Editorial de la Universidad del País Vasco. Bilbao.

Terrenato, N. 2001 Contexto, en Francovich, R. \& Manacorda D. Diccionario de Arqueología. 91-93. Crítica. Barcelona.
Terrenato, N. \& Ricci, G. 1998 I residui nella stratificazione urbana. Metodi di quantificazione e implicazioni per l'interpretazione delle sequenze: um caso di Studio dalle pendici settentrionali del Palatino, en Guidobaldi, F., Pavolini, C. \& Pergola, P. I materiali residui nello scavo archeologico. Testi preliminari e atti della tavola rotonda di Roma (16 marzo 1996). 89-104. Ecole Francaise de Rome. Roma.

Tuset, F. 1991 La Terra Sigillata de Clunia. Una propuesta metodológica para el estudio de las producciones altoimpreriales. Tesis Doctoral. Universitat de Barcelona. Barcelona.

Urteaga, M. M. \& Amundaray, M. L. 2003 Estudio de la cerámica procedente del puerto romano de Irún. Avance de las investigaciones. Boletín Arkeolan 11, 57-93.

Voss, B. \& Allen, R. 2010 Guide to ceramic MNV calculation qualitative and quantitative analysis. Technical Briefs in Historical Archaeology 5, 1-9. 